\title{
EXPECTATIVAS DEL PROGRAMA DE FORMACIÓN DOCENTE DE ADMINISTRACIÓN DE EMEPRESAS
}

\author{
EXPECTATIONS OF THE BUSINESS ADMINISTRATION TEACHER TRAINING PROGRAM \\ Referencia del artículo \\ Vides, R. (2020). COVID-19: Distanciamiento social, sus efectos psicológicos y 10 estrategias para sobrellevarlo. \\ Revista Científica del SEP. 3(1), 17-23. DOI: https://doi.org/10.36958/sep.v3i01.39
}

\author{
Mirza Yohana de León Lemus \\ yohana_lemus@hotmail.com \\ Licenciada en Administración de Negocios \\ Centro Universitario de Occidente CUNOC \\ Universidad de SanCarlos de Guatemala \\ Fecha de recibido: $30 / 06 / 2020$ \\ Fecha de aceptado: 03/11/2020
}

\begin{abstract}
RESUMEN
Según un estudio realizado por la Agencia de Estados Unidos para el Desarrollo Internacional (USAID):" El rol de los docentes en la mejora de la calidad educativa es prioritario, no será posible mejorar la calidad de la educación, si no se logra mejorar sustancialmente la calidad profesional de quienes se dedican a la enseñanza". (Vaillant, 2007).

Se pueden dar mejoras en varios aspectos que giran alrededor de la educación como la tecnologí-a y los ambientes, que definitivamente puede contribuir a mejorar la calidad de la educación, pero la clave está principalmente en el desempeño docente y ahí- es donde se prioriza la formación que los convierta en profesionales competentes.
\end{abstract}

Se presenta una investigación cualitativa que describe las expectativas docentes respecto al programa formativo en los primeros tres años de la carrera de Administración de Empresas del Centro Universitario de Occidente y asípresentar una propuesta educativa para contribuir al fortalecimiento de los procesos de capacitación y actualización.

Esta investigación identifica que la formación continua en los docentes es vital en su práctica, pues forma a un profesional que responde a las necesidades y expectativas de los estudiantes, pues busca e implementa alternativas que permiten mejorar los procesos de aprendizaje.

\section{PALABRAS CLAVE}

Expectativas, programa, formación, docente práctica

\section{ABSTRACT}

According to a study carried out by the United States Agency for International Development (USAID): "The role of teachers in improving educational quality is a priority, it will not be possible to improve the quality of education, if it is not possible to improve substantially the professional quality of those who are dedicated to teaching". (Vaillant, 2007).

Improvements can be made in various aspects that revolve around education such as technology and environments, which can definitely contribute to improving the quality of education, but the key is mainly in teacher performance and that is where the training that is prioritized is prioritized. make them competent professionals.

A qualitative research is presented that describes the teaching expectations regarding the training program in the first three years of the Business Administration career at the Centro Universitario de Occidente and thus present an educational proposal to contribute to the strengthening of the training and updating processes.

This research identifies that continuous training in teachers is vital in their practice, as it trains a professional who responds to the needs and expectations of students, as it seeks and implements alternatives that allow improving learning processes.

\section{KEYWORDS}

Expectations, program, training, teacher, practice 


\section{I.- INTRODUCCIÓN}

La investigación denominada "Expectativas del Programa de Formación Docente de la Carrera de Administración de Empresas", surge porque hoy se demanda una buena y adecuada práctica educativa, por lo que interesa conocer las expectativas docentes acerca del programa de formación y con ellas se proyecten líneas de acción en pro del adelanto de los profesionales.

(Vélaz de Medrano \& Vaillant, Aprendizaje y desarrollo profesional docente, 2009), menciona que la manera tradicional de enseñar ya no es útil, pues la sociedad y los estudiantes han cambiado. Se han ampliado los lugares para aprender, los sistemas para obtener información, la comunicación. Entonces es necesario que existan otros aportes en la manera de enseñar, por lo que se enfatiza una formación continua para todo docente.

Algo importante también es que (Vélaz de Medrano \& Vaillant, Aprendizaje y desarrollo profesional docente, 2009), menciona que los docentes interesan para lograr el aprendizaje en los estudiantes y con ello se mejore la calidad de la educación. Son un recurso necesario para la sociedad del conocimiento. Se necesitan buenas políticas para que la formación inicial de los docentes asegure las competencias que van a necesitar en su trayectoria profesional.

Se destaca también lo que dice (Davini, 2015), que para que se obtengan aprendizajes es a través de la capacitación, esto es, las acciones intencionales y planificadas que tienen como objetivo fortalecer los conocimientos, habilidades, actitudes y prácticas que tanto las escuelas como organizaciones no brindan, por lo menos en escala necesaria.

De tal manera que se da importancia a que un docente pueda seguir capacitándose o formándose, pues no debe estancarse y limitar su desarrollo profesional, es fundamental que su preparación sea constante.

Se desarrolló este tema por el interés de que al conocer las expectativas que se tienen respecto al Programa de Formación Docente, éstas se conviertan en guías de acción para conformar un proyecto que se enfoque en optimizar el desempeño docente.

En la carrera de Administración de Empresas hubo docentes que manifestaron que los contenidos abordados en el programa formativo docente sí responden a las necesidades que había. Esto está en relación con lo dicho por (Vélaz de Medrano \& Vaillant, Aprendizaje y desarrollo profesional docente, 2009), que mencionó que para formar a profesores debe hacerse en la capacidad de adaptación a un entorno cambiante y por eso resultan hoy elementos esenciales para orientar la formación docente.

Sin embargo, hubo docentes que consideraron que los contenidos abordados por parte del programa eran muy generales, creando así un desfase porque cada área posee necesidades específicas que se debían atender. Los docentes esperan que más que temas de capacitación existan temas de actualización, que se diera más énfasis en la parte pedagógica, que exista un equilibrio o hacer un estudio de cuáles son las áreas donde debe fortalecerse integralmente. Esto concuerda con lo que dice (Llena, París, \& Quinquer, 2003) , debe planificarse o diseñar qué se desea o qué se pretende y por dónde se quiere que vaya el proceso de aprendizaje.

Por lo tanto, se necesita la creación de una Comisión académica en la Carrera de Administración de Empresas para promover la creación de un seminario permanente de formación, actualización y autoformación docente para la carrera de Administración de Empresas valiéndose del programa de Formación Docente con el que cuenta El Centro Universitario de Occidente CUNOC.

OBJETIVO: Identificar las expectativas del docente respecto al programa formativo en los primeros tres años de la carrera de Administración de Empresas del Centro Universitario de Occidente.

METODOLOGÍA: Se realizó una investigación cualitativa, para conocer la percepción de los docentes del programa formativo en la carrera de Administración de Empresas del Centro Universitario de Occidente. Se utilizó el método fenomenológico, con guías recolectoras de datos semiestructuradas y de observación buscando entender las experiencias de docentes sobre el programa formativo, docentes de la carrera de y sus expectativas respecto a éste, utilizando una muestra en cadena o por redes de 15 y 2 autoridades: Coordinador del Programa Docente y el Coordinador de la carrera de Administración de Empresas.

RESULTADOS: La formación docente tiene su importancia en el proceso formador del estudiante. Es por ello por lo que el docente universitario debe tener una mejora continua en su trabajo adecuado a su contexto, pues de su actuar se crea una base que perciben los estudiantes y eso se tiende a imitar. Y cuando lo que se imita es bueno las cosas van por buen camino, pero si no es así se hace daño a más de una persona. Todo docente debe crear un perfil con características que respondan a las necesidades y expectativas que planteen sus estudiantes. 
Un docente no puede aseverar que no necesita la actualización en la formación específica que exige su actividad, pues los cambios en la sociedad son acelerados, existe diversificación en el saber pedagógico, la invasión de las nuevas tecnologías. (Santos Guerra, 2010, pág. 3). Es por ello que un docente debe formarse para prepararse dentro de varios ámbitos como el del conocimiento, actitudes, comportamientos y habilidades para desempeñarse eficazmente en su labor.

En el Centro Universitario de Occidente funciona un programa de Formación Docente que no es específico del Centro, sino que está establecido en el Campus Central como programa y que apoya la formación docente del CUNOC, por lo que la carrera de Administración de Empresas no cuenta con un programa de formación docente que sea propio de la carrera y no cuenta con una Comisión Académica que promueva la capacitación y actualización.

En el Campus Central se imparten cursos presenciales los cuales no pueden ser aprovechados por los docentes de CUNOC debido a distancia y horarios. También en el Campus Central se ha creado un programa Virtual en el que a distancia los docentes del CUNOC pueden tener acceso y llevar los cursos virtuales.

La participación de los docentes a los cursos que promueve el programa es diversa. A pesar de que ellos saben la importancia que tiene la formación docente, es su horario laboral el que no les permite asistir a todos los cursos del programa. Por ejemplo, si los cursos se programan para la mañana, no pueden asistir porque ya tienen compromisos en ese horario.

Un docente mencionó que se prepara para estudiar un doctorado en el extranjero y que debía prepararse en inglés básicamente para poder optar a ese doctorado, esto demuestra que mientras algunos docentes no dan tanta importancia a la preparación y actualización profesional, existen otros que sí tienen el deseo y la voluntad de hacerlo, pese a la dificultad de un idioma, pues reconocen el beneficio que eso reporta en su quehacer docente. Esto concuerda con lo dicho por (Vélaz de Medrano \& Vaillant, Aprendizaje y desarrollo profesional docente, pág. 36), que dijo que, el contexto social en que se ejerce la docencia es clave para tener un buen desempeño.

Sin embargo, hubo docentes que dijeron que todo docente que tuviera necesidades de formación que asistiera al programa de formación, es decir que no todos están interesados en asistir al programa de formación. $Y$ esto va muy relacionado a las razones por las que asisten algunos a los cursos del programa, por un lado, expresaron que la participación en los cursos estaba vinculada al ejemplo que debían dar ante las personas que tenía a su cargo, entonces en ese sentido se comprometía con los procesos de capacitación.

Por otro lado, expresaron su compromiso de poder avanzar y crecer como profesional y no para escalar un nivel sino de poder compartir y por ello desde que se inicia un curso lo finalizan como debe de ser. Esto concuerda con lo dicho por (Vaillant \& Marcelo, El ABC y D de la Formación Docente, 2015, pág. 20), que menciona que la responsabilidad es un factor personal y profesional que establece la capacidad de involucramiento y de aprendizaje de las personas, sobre todo en este siglo que vivimos.

Sin embargo, no concuerda con lo que expresaron otros docentes que manifestaron que el interés de obtener un diploma que les acredita, lo cual les sirve como créditos y es valioso para generar puntos con COMEVAL cuando les califican es lo que los mueve a asistir al programa formativo.

Además, hubo docentes que consideraron que los contenidos abordados por parte del programa son muy generales, pues se dan las mismas capacitaciones al área de Administración, Ciencia y Tecnología, Medicina y otras carreras, creando así un desfase porque cada área posee necesidades específicas que se deben atender.

Se espera que los cursos sean más sistematizados, pues no hay un programa que tenga seguimiento y que esté concatenado para poder desarrollar la labor docente, sería interesante que los cursos estuvieran en todo tiempo en todas las áreas.

Es necesario que se haga un sondeo de las necesidades que se tienen para propiciar en base a ellas los cursos a impartir.

Un dato importante del que se tuvo conocimiento fue que el Coordinador de la Carrera de Administración de Empresas manifestó saber del programa de Formación Docente de la USAC el cual se proyecta en el Centro Universitario de Occidente, pero un poco tarde, con lo que subraya una mala comunicación del funcionamiento de dicho programa, pues no sabía de cosas importantes como el solicitar al encargado del Programa alguna capacitación específica.

\section{2.- APORTE DEL PROGRAMA DE FORMACIÓN DOCENTE}

Los docentes de Administración de Empresas consideran que el Programa de Formación Docente ofrece un gran abanico de cursos para la formación docente, pero que necesitan la formación tecnológica para beneficiarse de ellos, pues existe el programa virtual en donde se pueden recibir talleres en línea, los cuales son interesantes, pero algunos no pueden llevarlos debido al poco conocimiento que tienen en cuanto al uso de la tecnología. 
Algo importante que se destaca por parte de los docentes, es que los programas contribuyen dependiendo de quién imparte los cursos y cómo lo hace. Según el testimonio de docentes ha habido charlas de profesionales cuyo desempeño ha sido pobre, pues las presentaciones son leídas y con poca participación de los docentes. Así también se han realizado eventos en donde el profesional se ha desempeñado satisfactoriamente.

El Coordinador del Programa de Formación Docente manifiesta que los cursos impartidos por el programa brindan un aporte a los docentes, pues temas como el medio ambiente son cuestiones de clima, cuidar el ambiente compete a todos; sin embargo, algunos lo ven como que no les compete a todos, no les interesa y por eso no lo reciben. La formación debe ser integral como profesionales, eso es lo que deberían reconocer los docentes que no asisten a los cursos.

Sin embargo, los docentes de Administración de Empresas manifestaron no estar interesados en cursos que no les beneficia en sí a su qué hacer universitario, pues no cuentan con mucho tiempo para su formación, por lo tanto, esperan que el programa sea más específico a su profesión y con ello se beneficien en su campo.

Los docentes esperan que se mejoren algunos temas, que se dé la parte pedagógica pues no se tiene a excepción de quienes tienen docencia universitaria. Que los cursos sean regulares, que sean talleres donde haya capacitación, inclusive donde se pueda contribuir y no que se impartan de manera aislada. Que los cursos no traten sobre filosofía de las instituciones o de cómo crear conciencia de lo que es la universidad, sino que sean temas que en verdad sean interesantes y puedan llevarse a la práctica.

\section{3.- HALLAZGOS IMPORTANTES}

Que los cursos que se imparten en la Universidad San Carlos de Guatemala, también se impartan en el Centro Universitario de Occidente. $Y$ que dichos cursos se impartan en horarios accesibles a los docentes para que puedan asistir.

Que el contenido de los cursos esté relacionado a su especialidad, así como no descuidar el nivel de los expositores de los cursos del Programa de Formación Docente.

Que todos los docentes asistan a los cursos del Programa de Formación Docente y contar con un Programa de Formación Docente específico para la División de Ciencias Económicas.

Que se realice un diagnóstico de necesidades para que los cursos del Programa de Formación Docente puedan cubrirlas.
Así como que existan más cursos de actualización que de capacitación en donde se de la sistematización, que haya un plan, un horario y se dé seguimiento.

Tener más personal que labore en el Programa de Formación Docente y que el horario sea por lo menos de 8 horas de oficina, que se cuente con un presupuesto para invitar a especialistas que impartan cursos específicos en el Programa de Formación Docente y que se impartan cursos para lograr formación pedagógica, que capacite sobre estrategias de aprendizaje, modelos de aprendizaje y se salga de la prueba objetiva.

\section{4.- CONCLUSIONES}

El Programa de Formación Docente no llena las expectativas del claustro de docentes, pues ellos tienen necesidades formativas que no se toman en cuenta, debido a que no se realiza un diagnóstico serio, objetivo y real de dichas necesidades, lo que conlleva a no planificar conferencias, talleres y actividades que correspondan a las carencias presentes y con ello se logre fortalecer esas áreas débiles que permitan al profesional mejorar su práctica docente.

Los docentes de la carrera de Administración de Empresas sin excepción de alguno, son convocados al programa de formación; sin embargo, no todos se hacen presentes debido a la carga académica que poseen, tienen el deseo de participar en algunos cursos de interés que promueve el programa, pero es una limitante sus múltiples actividades en la universidad.

El encargado del Programa de Formación Docente del Centro Universitario de Occidente, asiste 5 horas diarias a sus actividades, no cuenta con un horario de 8 horas para tener un horario de oficina y poder atender y realizar todo lo que el programa requiere, pues no solo se ve Formación Docente sino tiene que ver currículum, actualizaciones, autoevaluaciones que son cuestiones que no se logran abarcar del todo debido al horario reducido que se tiene de trabajo.

Las expectativas docentes respecto a ciertos profesionales que dan la capacitación y actualización en el programa de formación no se cumplen, más bien son decepcionantes porque las presentaciones son muy pobres ya que no tienen la parte pedagógica para impartir los cursos.

En la Universidad San Carlos de Guatemala se promueven cursos que son impartidos específicamente en el Campus Central, limitando a los docentes del Centro Universitario de Occidente a participar debido a distancia, gastos y horario. $\mathrm{Si}$ dichos cursos se impartieran en el CUNOC, podrían beneficiar a más profesionales si también se hicieran los esfuerzos por impartirlos a distancia. 


\section{5.- REFERENCIAS BIBLIOGRÁFICAS}

Davini, M. C. (2015). La Formación en la Práctica Docente. Buenos Aires: Paidós SAICF.

Llena, A., París, E., \& Quinquer, D. (2003). Planificación y análisis de la práctica educativa. España: Publidisa.

Pérez Porto, J., \& Merino, M. (Julián Pérez Porto y María Merino de 2012). Definición de expectativa - Qué es, Significado y Concepto. Obtenido de https://definicion.de/expectativa/

Santos Guerra, M. Á. (Agosto de 2010). La formación del profesorado en las instituciones que aprenden. Revista Interuniversitaria de Formación del Profesorado, pág. 3.

Vaillant, D. (2007). Pensamiento Educativo.

Vaillant, D., \& Marcelo, C. (2015). El ABC y D de la Formación Docente. Madrid: Narcea, S.A. DE EDICIONES.

Vázquez Travieso, R. L. (12 de mayo de 2017). La importancia de la capacitación docente. Obtenido de https: / /cea.uprrp.edu/la-capacitacion-docente-y-su importancia/

Vélaz de Medrano, C., \& Vaillant, D. (s.f.). En Aprendizaje y desarrollo profesional docente (págs. 21,22). España: METAS EDUCATIVAS.

Vélaz de Medrano, C., \& Vaillant, D. (2009). En Aprendizaje y desarrollo profesional docente. España: METAS EDUCATIVAS.

\section{Sobre autora}

\section{Mirza Yohana De León Lemus}

Es Administradora de Putificadora Brisas María (desde hace 5 años). Licenciada en Administración de Negocios con Postgrado en Recurso Humano de Universidad Galileo, Maestría en Docencia Universitaria por el Centro Universitario de Occidente CUNOC.

Copyright (c) 2020 Mirza Yohana de León Lemus

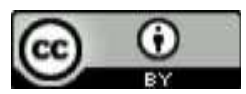

Este texto está protegido por una licencia Creative Commons 4.0.

Usted es libre para Compartir - copiar y redistribuir el material en cualquier medio o formato - y Adaptar eldocumento - remezclar, transformar y crear a partir del material- para cualquier propósito, incluso comercialmente, siempre que cumpla la condición de:

Atribución: Usted debe reconocer el crédito de una obra de manera adecuada, proporcionar un enlace a la licencia,e indicar si se han realizado cambios. Puede hacerlo en cualquier forma razonable, pero no de forma tal que sugieraque tiene el apoyo del licenciante o lo recibe por el uso que hace.

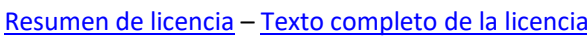

